\title{
Impact of GC-MS in Analysis of Cancer Biomarkers
}

\author{
Sharib Raza Khan, Hobbs Bower Reang and Rohit Bhatia* \\ Department of Pharmaceutical Analysis, ISF College of Pharmacy, India \\ *Corresponding author: Rohit Bhatia, Department of Pharmaceutical Analysis, ISF College of Pharmacy, India
}

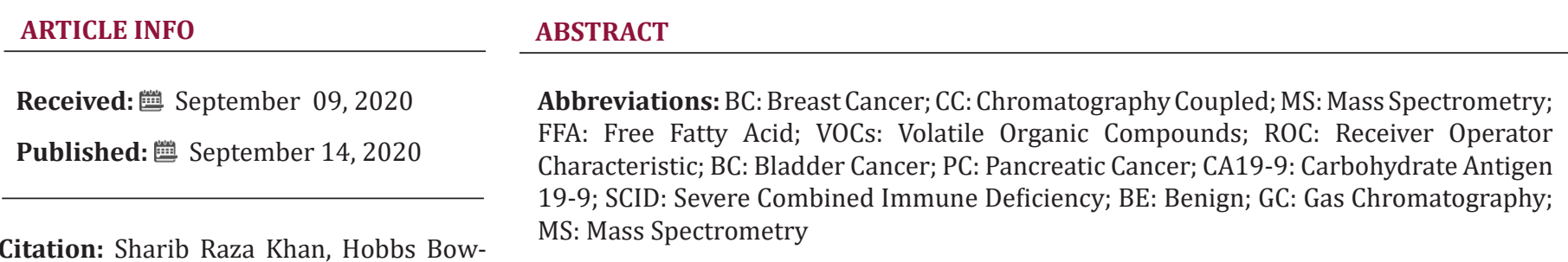

er Reang, Rohit Bhatia. Impact of GC-MS in Analysis of Cancer Biomarkers. Biomed J Sci \& Tech Res 30(3)-2020. BJSTR. MS.ID.004942.

\section{Editorial}

Cancer is the one of the most dreadful diseases of the modern age. A huge number of chemical biomarkers are associated with progression and abnormalities associated with variety of cancers. Therefore detection of these biomarkers in various biological fluid is an significant approach to detect the stage and pathophysiology of cancer. Several analytical reports are available in literature where researchers have utilized different analytical tools for determination of cancer biomarkers. Gas Chromatography Coupled with Mass Spectrometry (GC-MS) in one of the advanced techniques which has been successfully utilized for detection and quantification of cancer biomarkers in past few years. In the following sections of this compilation we have highlighted some significant reports revealing impact of GC-MS technology in detection of cancer biomarkers in biological fluids.

The abnormal free fatty acid metabolism corresponds to Breast Cancer (BC). Therefore an accurate and precise method was investigated for identification of serum Free Fatty Acid (FFA) metabolic profiles and screening of potential biomarkers for breast cancer diagnosis utilizing the GC-MS technique. The serum Free Fatty Acid (FFA) as potential biomarkers were identified and screened by gas chromatography-mass spectrometry and the results of total eighteen free fatty acids in serum with the VIP threshold value $(>1)$ in the OPLS-DA model as well as the FDRcontrolled student's t-test ( $p$-value $<0.1$ ), also the results of five

potential serum biomarker with AUC $(>0.75)$ with $p$-value $(<0.01)$ were determined by this technique. This method was advantageous for clinical non-invasive diagnosis of breast cancer and inform further mechanistic investigation in breast cancer [1].

Volatile Organic Compounds (VOCs) include endogenous metabolites that provide information about health and disease which might be useful to develop a better screening method for breast cancer. Gas Chromatography-Mass Spectrometry QTOF approach was utilized to detect VOCs in urine by preparing the samples utilizing solid phase micro-extraction. A set of six VOCs classifed mice with and without tumors with an area under the Receiver Operator Characteristic (ROC AUC) of 0.98 via fivefold cross validation. This study revealed that not only do VOCs change due to an alteration in metabolism (cancer/no cancer model), but it also shows unique VOCs released by specific tumormicroenvironment interactions (localized/metastasized model) [2]. Bladder cancer (BC) is one of the seventh most common cancers in UK and the chances of survival are greatly increased by early diagnosis. Therefore a non-invasive, accurate, rapid and cheap was investigated for the identification of bladder cancer by Volatile Organic Compounds (VOCs) present in urine headspace as diagnostic biomarkers for bladder cancer by utilizing the GC-MS technique. Cystoscopy with biopsy and urine cytology technique was used earlier as a diagnostic tool for bladder cancer detection. 
The GC-MS technique was successfully utilized to examine this hypothesis by urine samples collected from diagnosed bladder cancer patients and from healthy volunteers, from which the volatiles metabolomes were analysed. The result obtained by independent test data provided by either both patients was $89 \%$ overall accuracy, $90 \%$ sensitivity and $88 \%$ specificity. This method is advantageous and considerably better than the earlier method and further improvement is still highly warranted [3].

Pancreatic Cancer (PC) is a lethal solid tumour which is one of the leading causes of cancer-related death. Therefore a novel, accurate and precise screening method has been investigated by many researchers for biomarker development that would enable diagnosis of pancreatic cancer by utilizing the GC-MS/ MS technique. Carbohydrate Antigen 19-9 (CA19-9), as a tumour marker for PC and screening methods such as magnetic resonance imaging, computed tomography, and endoscopic ultrasonography was used as a diagnostic tool for pancreatic cancer detection. The GC-MS/MS based targeted metabolomics and multivariate logistic regression analysis technique has been successfully utilized to identify potential biomarkers by blood samples collected from two independent groups' i.e. pancreatic cancer patients and healthy volunteers. The results obtained by multivariate test provided by either both group patients revealed very high sensitivity $100 \%$ for resectable PC than CA19-9. This method was highly accurate and better in finding the prognosis of pancreatic cancer in patients by aiding early detection of the disease [4].

Gastric cancer is one of the second most common cancer worldwide and leading cause of death in Asian countries such as China and India. Currently, endoscopy and pathological examinations used as a diagnostic tool for gastric cancer detection. Consequently, a convenient and non-invasive approach to identify biomarkers in urine especially for recurrent gastric cancer was developed utilizing GC-MS technique. Due to high sensitivity, reproducibility and peak resolution GC-MS has been widely used in metabolomics study. In this study, a human gastric non-metastasis model for cancer and a metastasis mice model with a Severe Combined Immune Deficiency (SCID), and GC-MS was deployed following chemical derivative to profile urinary specimens of the mouse model and its urine matched. The result of metabolic differences among three groups ( $\mathrm{t}$ test, $\mathrm{P}<0.05$ ) was significant and metastasis was characterized respectively by the Principal Component Analysis (PCA). These PCA models have been confirmed by corresponding analysis of the operating characteristics of the receiver (Area under Curve = 1.00). This method was accurate, instructive and helpful in clinical diagnosis or screening metastasis for gastric cancer [5]. GC-MS approach was utilized to investigate the Free Fatty Acid (FFA) metabolic profiles and to identify biomarkers that can be used to distinguish patients with Breast Cancer (BC) from Benign (BE) patients or healthy controls. Three saturated fatty acids (C14:0,
C16:0 and C18:0) and three unsaturated fatty acids (C18:2, C18:3 and C20:5) in BC were significantly different than controls. Palmitic acid, stearic acid, linoleic acid and total FFA were identified as potential biomarkers distinguished $\mathrm{BC}$ from the other groups. The alterations of FFA could reflect underlying metabolic changes in BC patients, and this study has demonstrated that FFA biomarkers might be helpful for prevention and characterization of BC patients [6].

Among the various techniques conventionally available, GCMS is a powerful hyphenated technique for identification and quantification of metabolites particularly, plasma fatty acids for breast cancer due to its high sensitivity, peak resolution and reproducibility. In another study GC-MS was utilized to identify serum free fatty acid (FFA) metabolic profiles and screen potential biomarkers for BC diagnosis. GC-MS and in-house fatty acid methyl ester standard substances library were combined to accurately identify FFA profiles in serum samples of BC patients and breast adenosis patients (as controls). Potential biomarkers were screened by applying statistical analysis. A total of eighteen free fatty acids were accurately identified in serum sample. This method was useful in the analysis of free fatty acid profiles and a powerful tool for the prevention and characterisation of $\mathrm{BC}$ patients as well as the elucidation of disease mechanisms, the screening of novel biomarkers and the monitoring of clinical pharmacological therapy [7]. GC-MS technique followed by chemometric analysis to obtain metabolic profiles for the prediction of BC diagnosis, grading, staging and neoadjuvant status by collecting serum samples from pre-operative Breast Cancer (BC) patients and healthy controls was successfully utilized. Human gastric cancer SGC-7901 cells were implanted into 24 Severe Combined Immune Deficiency (SCID) mice, which were randomly divided into metastasis group $(n=8)$, non-metastasis group ( $\mathrm{n}=8)$, and normal group ( $\mathrm{n}=8)$. Urinary metabolomic information was obtained by Gas Chromatography/ Mass Spectrometry (GC/MS). Seven metabolites have been selected, which constructed a diagnostic model for distinguishing the nonmetastatic and the metastatic gastric cancer [8].

In another study, an attempt was made to classify mice with and without tumours and compare tumours localized to the mammary pad and tumour cells injected into the iliac artery by differences in VOCs in urine. The results were analysed by gas chromatographymass spectrometry quadrupole time-of-flight with solid phase microextraction. To find potential biomarkers for breast cancer and metastatic breast cancer in mice models, multivariate and univariate statistical analyses were employed. A set of six VOCs classified mice with and without tumours with an area of 0.98 (95 percent confidence interval $[0.85,1.00])$ below the Receiver Operator Characteristic (ROC AUC) via five-fold cross validation. Mice with tumours in the mammary pad and iliac artery were classified using a different set of six VOCs with 0.96 ROC AUC (95\% confidence 
interval $[0.75,1.00])$. This method was simple, sensitive and less invasive and helpful in developing a better screening method for breast cancer [9]. The above reports evidenced the success story of GC-MS in cancer biomarker identification/determination any many research groups are continuously exploring this technique for the same. This compilation might be useful for the analysts/clinicians to get insight into the utility of GC-MS technique.

\section{References}

1. Józ J, Kału J (2014) Current applications of chromatographic methods for diagnosis and identification of potential biomarkers in cancer. Trends in Analytical Chemistry 56: 1-12.

2. Rajak H, Deshmukh R, Aggarwal N, Kashaw S, Kharya MD, et al. (2009) Synthesis of novel 2,5-disubstituted 1,3,4-thiadiazoles for their potential anticonvulsant activity: Pharmacophoric model studies. Arch Pharm (Weinheim) 342(8): 453-461.

3. Cauchi M, Weber CM, Bolt BJ, Spratt PB, Bessant C, et al. (2016) Analytical Methods spectrometry and pattern recognition for the identi fi cation of bladder cancer from urine headspace pp. 4037-4046.

\section{ISSN: 2574-1241}

DOI: $10.26717 /$ BJSTR.2020.30.004942

Rohit Bhatia. Biomed J Sci \& Tech Res

(C) (P) This work is licensed under Creative

Submission Link: https://biomedres.us/submit-manuscript.php
4. Lubes G, Goodarzi M (2018) GC-MS based metabolomics used for the identification of cancer volatile organic compounds as biomarkers. J Pharm Biomed Anal [Internet] 147(5): 313-322.

5. Hirata Y, Kobayashi T, Nishiumi S, Yamanaka K, Nakagawa T, et al. (2017) Identification of highly sensitive biomarkers that can aid the early detection of pancreatic cancer using GC/MS/MS-based targeted metabolomics Clin Chim Acta [Internet] 468: 98-104.

6. Lv W, Yang T (2012) Identi fi cation of possible biomarkers for breast cancer from free fatty acid pro fi les determined by GC - MS and multivariate statistical analysis. Clin Biochem [Internet] 45(1-2): 127133.

7. Ang CY, Tan SY, Zhao Y (2014) Recent advances in biocompatible nanocarriers for delivery of chemotherapeutic cargoes towards cancer therapy. Org Biomol Chem 12(27): 4776-4806.

8. Hu J, Tang H, Zhang Q, Fan J, Hong J, et al. (2011) Prediction of gastric cancer metastasis through urinary metabolomic investigation using GC / MS. World J Gastroenterol 17(6): 727-734.

9. Hadi NI, Jamal Q, Iqbal A, Shaikh F, Somroo S (2017) Serum Metabolomic Profiles for Breast Cancer Diagnosis, Grading and Staging by Gas Chromatography-Mass Spectrometry. Sci Rep [Internet] pp. 1-11.

$\begin{array}{ll}\text { BIOMEDICAL } & \text { Assets of Publishing with us } \\ \text { RESEARCHES } & \text { - Global archiving of articles } \\ \text { - Immediate, unrestricted online access } & \text { - Rigorous Peer Review Process } \\ & \text { - Authors Retain Copyrights } \\ & \end{array}$

